\title{
Prevalence of Non-Alcoholic Fatty Liver with Gall Stones Disease in Himalayan Population
}

\author{
Ankit Sharma ${ }^{1}$, Kunal Malhotra ${ }^{2}$, Ashok Kumar Kaundal ${ }^{3}$, Anil Malhotra ${ }^{4}$ \\ 1Department of Surgery, Indira Gandhi College and Hospital, Shimla, Himachal Pradesh, India. \\ ${ }^{2}$ Department of Surgery, Indira Gandhi College and Hospital, Shimla, Himachal Pradesh, India. \\ ${ }^{3}$ Department of Surgery, Indira Gandhi College and Hospital, Shimla, Himachal Pradesh, India. \\ ${ }^{4}$ Department of Surgery, Indira Gandhi College and Hospital, Shimla, Himachal Pradesh, India.
}

\section{ABSTRACT}

\section{BACKGROUND}

Non-Alcoholic Fatty Liver Disease (NAFLD) is the hepatic pandemic of the $21^{\text {st }}$ century. It is the amassing of fats in the hepatic tissue without significant alcohol intake that results in hepatic steatosis. Patients with gall bladder stones may have associated NAFLD as these ailments share similar factors like obesity, hypertriglyceridemia and diabetes mellitus. However, few, if any, reports are available about the association of NAFLD with gallstones in the hilly population. Hence, this study was conducted to find out the prevalence of NAFLD in patients with gall bladder stone disease.

\section{METHODS}

This study was done in the Department of Surgery, Indira Gandhi Medical College, Shimla, from June 2017 to May 2019. A total of 300 patients of ultrasound proven gall bladder stones was studied for NAFLD by Fibroscan (transient elastography). Transient elastography (TE) is a noninvasive method that has been shown to be useful for the detection of liver steatosis and fibrosis. NAFLD was diagnosed based on the value of CAP (Controlled Attenuation Parameter) \& degree of fibrosis was assessed based on liver stiffness measurement (LSM) value on TE. Steatosis was graded as S0, S1, S2, and S3 while fibrosis was graded as F0-F1, F1, F2, F3, and cirrhosis. Minimum cut-off CAP value for diagnosing NAFLD was $214 \mathrm{~dB} / \mathrm{m}$ \& significant fibrosis was taken with LSM value $>7.5 \mathrm{kPa}$.

\section{RESULTS}

Patients of gall stone disease showed significant liver steatosis, suggestive of NAFLD in 189 patients (63\%), based on CAP value; however, 111 patients $(37 \%)$ did not have significant steatosis. In patients with NAFLD, 57 (30\%) had mild steatosis (s1) while $39(20.53 \%) \& 24(12.63 \%)$ had moderate (s2) and severe (s3) steatosis respectively. Similarity, 72 (24\%) patients had significant fibrosis while 228 (76\%) patients had no to insignificant fibrosis on TE, $51(17 \%)$ patients had moderate fibrosis, while $14(4.5 \%)$ and 8 (2.5\%) patients had severe fibrosis \& cirrhosis respectively.

\section{CONCLUSIONS}

High prevalence of NAFLD in patients of gall stone disease was observed. Most of the patients had mild NAFLD i.e. grade S1 steatosis \& in addition, fibrosis was present in $24 \%$ patients of NAFLD with gall stone disease.

\section{KEY WORDS}

Gall Stone Disease (GSD), Non-Alcoholic Fatty Liver Disease (NAFLD), Steatosis, Fibrosis, Transient Elastography (TE)
Corresponding Author:

Dr. Kunal Malhotra,

Kushal Kunj,

Near JCB Public School, Sector-3,

New Shimla, Shimla-171009,

Himachal Pradesh, India.

E-mail: anilkushalmalhotra@gmail.com

DOI: $10.14260 /$ jemds/2020/201

Financial or Other Competing Interests: None.

How to Cite This Article:

Sharma A, Malhotra K, Kaundal AK, et al. Prevalence of non-alcoholic fatty liver with gall stones disease in Himalayan population. J. Evolution Med. Dent. Sci. 2020;9(12):932-936,

$10.14260 / \mathrm{jemds} / 2020 / 201$

Submission 13-01-2020,

Peer Review 27-02-2020,

Acceptance 04-03-2020,

Published 23-03-2020.

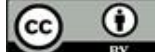




\section{BACKGROUND}

Gallstones constitute a significant health problem affecting 10 - $15 \%$ of the adult population \& non-alcoholic fatty liver disease (NAFLD) is the hepatic pandemic of the $21^{\text {st }}$ century $\&$ is the number one cause of chronic hepatic disease.(1) Patients with gallstones may have associated NAFLD as these ailments share similar factors for example obesity, hypertriglyceridemia and diabetes mellitus. There is a high prevalence of gall stone disease among patients with NAFLD versus those without NAFLD \& NAFLD being an independent risk factor for patients with GSD. Moreover, GSD prevalence increased with advancing fibrosis and severity of necroinflammatory activity. Thus, NAFLD is associated with a high prevalence of GSD, which would characterise patients with more advanced liver disease. And NAFLD and GSD are significantly associated in a bidirectional fashion. Thus, although NAFLD appears to be an independent risk factor for GSD, the latter represents an independent risk factor of NAFLD. (2)

Although the gold standard for diagnosis and staging NAFLD is liver biopsy. Liver biopsy has inherent limitations because of risks and life threatening complications, sample size and sampling variability which render liver biopsy a less than ideal gold standard.(3) Ultrasound is commonly used to screen for hepatic steatosis, but its limitations include suboptimal sensitivity as well as specificity for the detection of mild steatosis \& operator dependence. Hence it is not ideal for detecting the early stages of NAFLD.(4) Transient elastography (fibroscan) is a new, user friendly, noninvasive test. TE for the evaluation of cirrhosis has shown validity and excellent diagnostic accuracy in chronic hepatitis C \& B, alcoholic liver disease, primary biliary cholangitis and after liver transplant. It has high sensitivity and specificity in mild to moderate steatosis and fibrosis. (5)

Thus, this study is possibly the first attempt to study the prevalence of NAFLD in patients with gall stone disease by using transient elastography in Himalayan population of the Indian subcontinent, which represents a distinct geographic, environmental and diet consuming entity than the rest of the Indian subcontinent.

\section{METHODS}

This cross-sectional study was done in the department of surgery from June 2017 to May 2019 in Tertiary care medical college hospital in hill state of Himachal Pradesh which is a part of western Himalaya. And patients from all over the state are being referred \& treated in this hospital. A total of 300 patients of ultrasound proven cholelithiasis admitted in hospital for cholecystectomy, underwent Fibroscan in the department of Gastroenterology and were evaluated to establish relationship between cholelithiasis and NAFLD.

\section{Inclusion Criteria}

Were aged 18 years or above, diagnosed gall bladder stone disease on screening ultrasonography for right hypochondriac pain or biliary pain who underwent cholecystectomy.

\section{Exclusion Criteria}

Were clinical, radiological or biochemical evidence of choledocholithiasis, evidence of gall stone induced pancreatitis, pregnancy, patients with significant alcohol addiction $>25 \mathrm{~g} /$ day, patients not willing to participate or on hepatotoxic drugs.

\section{Data Collection}

Data was recorded and baseline demographic data of all study patients including - Age, Sex, height, weight, BMI (body mass index), blood pressure were recorded. GPE (general physical examination) and systemic examination was done. All routine and relevant investigations to exclude secondary causes of fatty liver including estimation of blood glucose, HbA1c, complete hemogram, renal function tests, ALT, AST, total and direct bilirubin, CRP, thyroid function tests, total protein and albumin and lipid profile, Hep B, Hep C., HIV serology, serum ceruloplasmin and ANA were done. The patients with investigations suggestive of alternative diagnosis were excluded.

The lab. investigations included estimation of blood glucose, HbA1c, complete hemogram, renal function tests, SGOT, SGPT, total and direct bilirubin, CRP, thyroid function tests, total serum proteins, serum albumin and lipid profile. Abdominal USG examination of the subjects was performed using a $3.5 \mathrm{MHz}$ transducer and the presence of gallstones was determined.

NAFLD was diagnosed based on value of CAP (controlled attenuation parameter) on transient elastography (fibroscan), simultaneously degree of fibrosis was assessed based on liver stiffness measurement (LSM) value on transient elastography. Based on CAP value steatosis was graded as S0, S1, S2, S3 and fibrosis was graded as, F0-F1, F2, F3 and cirrhosis. Minimum cut-off value for diagnosing NAFLD was $214 \mathrm{~dB} / \mathrm{m}$. Minimum fibrosis significant was taken as F2 or more i.e. LSM value $>7.5 \mathrm{kPa}$.

The finding of Fibroscan were interpreted as: S0 (no fatty liver), $S \geq 1$ ( $\geq 10 \%$ of hepatocytes with fat), the CAP cut-off values ranged from 214 to $289 \mathrm{~dB} / \mathrm{m}$, with a $64 \%-91 \%$ sensitivity and $64 \%-94 \%$ specificity; for $\mathrm{S} \geq 2(\geq 33 \%$ hepatocytes with fat), the CAP cut-off values ranged from 255 to $311 \mathrm{~dB} / \mathrm{m}$, with a $57 \%-96 \%$ sensitivity and $62 \%-94 \%$ specificity; finally, for S3 ( $\geq 66 \%$ hepatocytes with fat), the CAP cut-off values ranged from 281 to $310 \mathrm{~dB} / \mathrm{m}$ with a $64 \%$ $100 \%$ sensitivity and $53 \%-92 \%$ specificity. S1, S2, S3 were graded as mild, moderate and severe NAFLD respectively. The adjusted values were taken as: S0, if CAP score is $<214$, S1, if CAP score is (215-255), S2, if CAP score is (256-312), and S3, if CAP score is $>312 \mathrm{~dB} / \mathrm{m}$. And F0-F1 i.e. no to mild fibrosis if LSM score was between 2.5 and $7.5 \mathrm{kPa}, \mathrm{F} 2$ i.e. moderate fibrosis if LSM score was between 7.5- $10 \mathrm{kPa}, \mathrm{F} 3$ i.e. severe fibrosis if LSM score was between $10-14 \mathrm{kPa}$ and F4 i.e. cirrhosis if LSM score is $>14 \mathrm{kPa}$. Significant fibrosis was taken as F2 and above.

\section{Statistical Analysis}

All numerical data were expressed as mean with standard deviation (SD). All the statistical tests were done using Excel/SPSS software. Discrete and continuous variables were compared using Pearson's coefficient, Chi square test and Student t-test as appropriate. Multiple comparisons were 
made using ANOVA. A p value $<0.05$ was taken as statistically significant.

\section{RESULTS}

This study, was conducted from 1st of June 2017 to May 31st 2019, included 300 patients who came to surgery or gastroenterology OPD with ultrasonography proven cholelithiasis (gall stone disease). Out of 300 patients in the study $240(80 \%)$ patients were females and $60(20 \%)$ were male respectively. Patient's ranged from 22 to 80 years of age with an average age of 44.3 yrs. (SD-11.9). Maximum numbers of patients were in the age group of 31-50 yrs. constituting $57.5 \%$ of the total study population. Age wise distribution showed 43 patients (14.3\%) in age group 18-30 years, 76 (25.3\%) patients in age group 31-40 yrs., 96 (32\%) patients in age group 41-50 yrs., $51(17 \%)$ patients in age group 51-60 yrs., 30 (10\%) patients in age group 61-70 yrs., and $4(1.4 \%)$ patients in age group $>70$ years.

The mean BMI of the study population was 25.7(SD-3.7), with a range from 16.5 to 36.3. Mean LFT's of the study population were found to be within normal limits, in which, mean total bilirubin of the study population was $0.77 \mathrm{mg}$ $\%$ (SD-0.5), with a range from $0.1 \mathrm{mg} \%$ to $4.8 \mathrm{mg} \%$, and mean conjugated bilirubin was $0.24 \mathrm{mg} \%$ (SD 0.20 ), with a range from $0.01 \mathrm{mg} \%$ to $0.96 \mathrm{mg} \%$. Mean ALT of the study population was $34.9 \mathrm{U} / \mathrm{L}(\mathrm{SD}-16.7)$, with a range from $10 \mathrm{U} / \mathrm{L}$ to $111 \mathrm{U} / \mathrm{L}$. Mean AST of the study population was 33.5 U/L(SD-20.1), with a range from $15 \mathrm{U} / \mathrm{L}$ to $205 \mathrm{U} / \mathrm{L}$. Mean ALP of the study population was 103.4 U/L (SD-45.5) with a range from $43 \mathrm{U} / \mathrm{L}$ to $370 \mathrm{U} / \mathrm{L}$.

Mean total cholesterol of the study population was 179.3 $\mathrm{mg} / \mathrm{dL}$ (SD-40.5) with a range from $100 \mathrm{mg} / \mathrm{dL}$ to 287 $\mathrm{mg} / \mathrm{dL}$. Mean triglycerides of the study population were 71.6 $\mathrm{mg} / \mathrm{dL}$ (SD-71.6), with a range from $32 \mathrm{mg} / \mathrm{dL}$ to $474 \mathrm{mg} / \mathrm{dL}$. Mean HDL of the study population was $48.8 \mathrm{mg} / \mathrm{dL}$ (SD-13.1), with a range from $24 \mathrm{mg} / \mathrm{dL}$ to $119 \mathrm{mg} / \mathrm{dL}$. (table-1) In this study, significant liver steatosis, suggestive of NAFLD was found in 189 patients, i.e. $63 \%$, however 111 patients i.e. $37 \%$ did not have significant steatosis suggestive of NAFLD, calculated on the basis of CAP value. Those with significant steatosis suggestive of NAFLD were graded as $90(30 \%)$ mild steatosis (S1), 62(20.6\%) moderate steatosis (S2) and 37 $(12.4 \%)$ severe steatosis (S3). Similarly, fibrosis was calculated on the basis of LSM score and it was found that 79 patients, i.e. $26.3 \%$ had significant fibrosis (basic cut-off value for significant fibrosis was taken as $>7.5 \mathrm{kPa}$ ) and 221 patients did not have significant fibrosis.

\begin{tabular}{|cccc|}
\hline Variable & $\begin{array}{c}\text { GSD without NAFLD } \\
\text { (Mean) }\end{array}$ & $\begin{array}{c}\text { GSD with NAFLD } \\
\text { (Mean) }\end{array}$ & p-Value \\
Age & 43.5 & 44.8 & 0.43 \\
TOT. Bilirubin & 0.76 & 0.77 & 0.538 \\
Conj. Bilirubin & 0.27 & 0.22 & 0.335 \\
ALT & 31.4 & 37.0 & 0.018 \\
AST & 30.3 & 35.5 & 0.143 \\
ALP & 105.0 & 102.4 & 0.431 \\
TG & 139.2 & 180.0 & $<0.001$ \\
HDL & 49.6 & 48.3 & 0.499 \\
LDL & 97.6 & 105.7 & 0.086 \\
Total Cholesterol & 169.2 & 185.4 & 0.005 \\
Steatosis & 175.3 & 271.5 & $<0.001$ \\
Fibrosis & 4.3 & 7.8 & $<0.001$ \\
\hline \multicolumn{4}{r|}{ Table 1. Comparison of Various Parameters in } \\
Gall Stones Patients with and without NAFLD \\
\hline \multicolumn{4}{c}{} \\
\hline
\end{tabular}
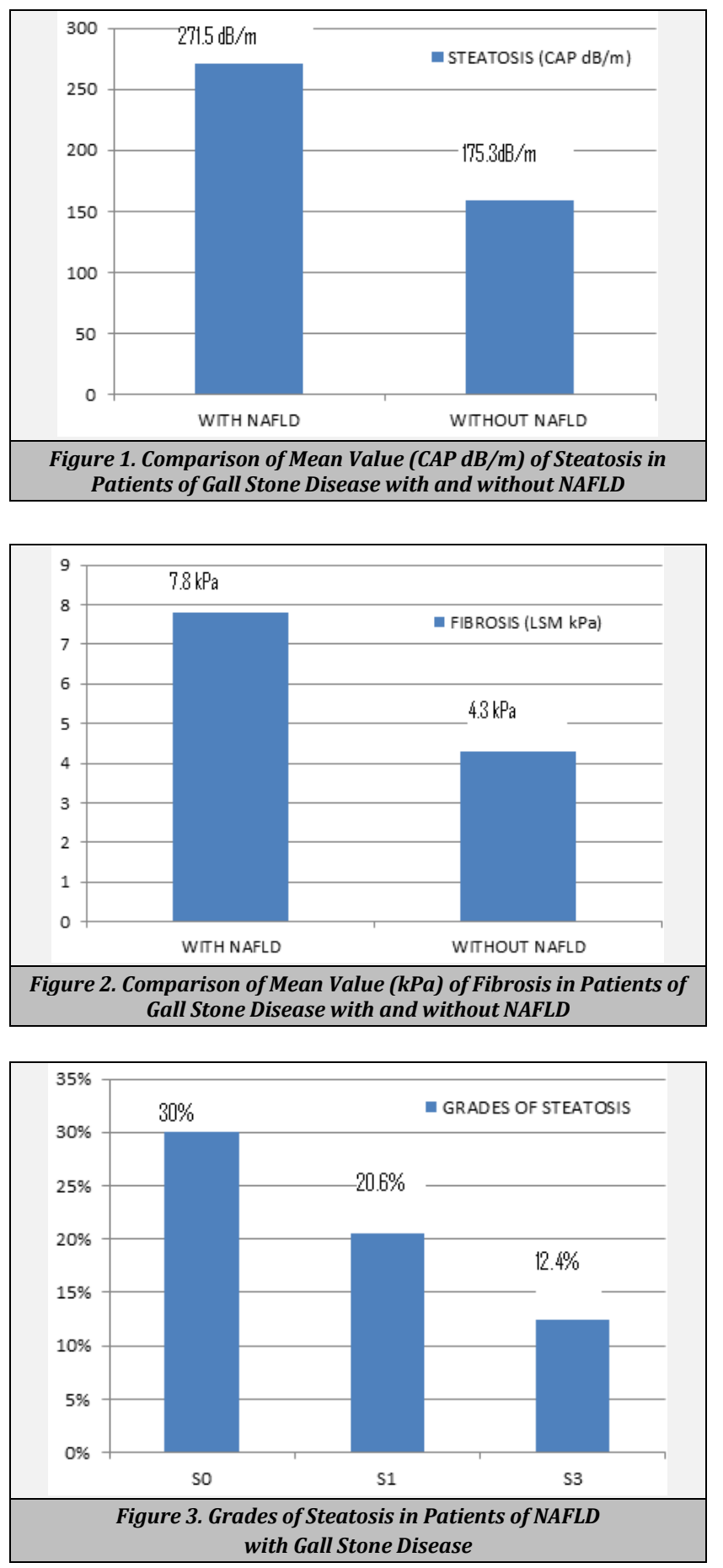

Fibrosis was graded as F0-F1, F2, F3 and cirrhosis i.e. noto insignificant fibrosis, moderate fibrosis, severe fibrosis and cirrhosis, based on the cut-off values and out of 300 patients, $221(73.7 \%)$ patients had no to insignificant fibrosis(f0-f1), $51(17 \%)$ patients had moderate fibrosis, 14 (4.6\%) patients had severe fibrosis and $7(2.4 \%)$ patients had cirrhosis on TE (fibroscan). In this study, patients of gall stone disease with NAFLD and patients without NAFLD were compared and it was found that: Mean age of patients with NAFLD was found to be 44.8 yrs., and without NAFLD was 43.5 yrs. with a pvalue of 0.43 . Thus, no significant association was found between age and NAFLD in patients with gall stone disease. Mean number of male patients, with NAFLD were 80.7 and without NAFLD were 79.0. Similarly, the mean number of females with NAFLD were 19.3 and without NAFLD were 21.0 , with a p-value of 0.771 . Thus, no significant association 
was found between sex and NAFLD in patients with gall stone disease.

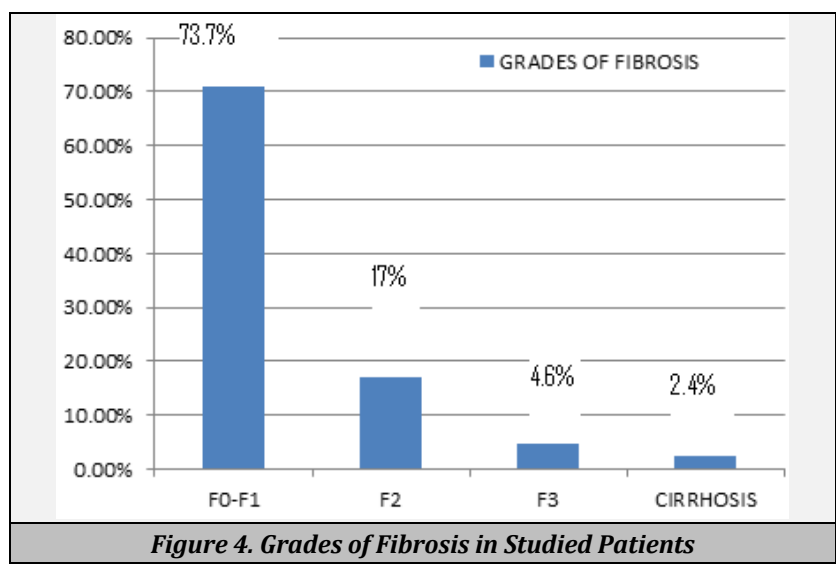

\section{DISCUSSION}

In our study, most of the patients were females (80\%), with male: female $=4: 1$, most of the patients belonged to age group 41 to $50 y$ rs (37\%), with mean age of $44.3 y$ rs. Other studies have also shown the same trend and reported more prevalence of gall stone disease in females as compared to males. Shaffer et al.[1] And Tsai et al[6] have concluded that age $>40$, and the female sex are regarded as major risk factors for the development of cholesterol cholelithiasis. A nationwide survey conducted in America revealed a very high prevalence of cholelithiasis i.e. $13.9 \%-26.7 \%$ in women and $5.3 \%-8.9 \%$ in men under the domain of third National Health and Nutrition Examination Survey.[7] However in India, prevalence of gall stones is $5.59 \%$ in women and $1.99 \%$ in males. There is a general conjecture that sex hormones and cholesterol metabolism have possible interrelation. ${ }^{[3]}$ This makes a point to ruminate on how sex is implicated in cholesterol stones formation. Most of the studies suggest female predominance when it comes to the prevalence of gallstones along with it the likelihood of being at risk for stone formation. $[1,2]$

Non-alcoholic fatty liver disease (NAFLD) has a prevalence of $15 \%-20 \%$ in the general population.[6] In our study NAFLD as detected by CAP value on TE, was present in $63 \%$ patients of GSD. The higher prevalence of NAFLD in our patients than the general population, suggest a possible association between NAFLD and gall stone disease. Similar higher prevalence of NAFLD has been reported in other studies which are in consonance with this study. Koller et al [8] found higher prevalence of GSD among patients with NAFLD versus those without NAFLD (47\% vs. 26\%, respectively).While, Farah et al from Pakistan reported the prevalence of NAFLD in $62.5 \%$ patients with gall stones disease. ${ }^{[9]}$ Higher prevalence of NAFLD in patients with gall stone disease is due to sharing of common risk factors like body mass index, gender, raised lipid levels, use of contraceptives, alcohol and having diabetes, physical inactiveness, multiparous women, water with excessive iron content \& metabolic syndrome.[10]

Most of the patients in our study population had mild severity of steatosis (S1 i.e. 30\%), this is in contrast to other studies which found that there is a greater degree of steatosis among patients with NAFLD and gallstones. Francazani et al.[2] including 524 Italian patients with biopsy-proven NAFLD found a GSD prevalence of $20 \%$ with NASH prevalence being significantly higher in patients with GSD, as compared to patients without GSD ( $77 \%$ vs. $56 \%$ ).

In our study, we found that fibrosis was present in $26.5 \%$ of patients and the mean fibrosis score was higher $(7.8 \mathrm{kPa})$ in patients with NAFLD than those without NAFLD (4.3 kPa) with a significant $p$-value of $<0.001$. Thus, we come to a result that fibrosis present in GSD with NAFLD is severe as compared to gall stone disease patients without NAFLD. The result of one series showed about $10 \%$ of the gallstone patients at the time of diagnosis had NAFLD progression to fibrosis which was biopsy proven. NAFLD contributes as the commonest cause that leads to cirrhosis, portal hypertension and hepatic cancer and it has been estimated that approximately $22 \%$ of NAFLD progresses to cirrhosis [10, 11], One of the studies justified for performing liver biopsy for the detection of NAFLD during cholecystectomy and that series captured more NAFLD in patients who undergone biopsies than those with simple ultrasound findings and liver function tests.[12]

\section{CONCLUSIONS}

There is a high prevalence of NAFLD in patients of gall stone disease in Himalayan population. Most of the patients of NAFLD with gall stones had mild degrees of steatosis. Fibrosis was present in $27 \%$ patients of NAFLD with gall stone disease. Patients of gall stone disease with NAFLD had a higher mean BMI, TG, T. Ch, steatosis and fibrosis levels than gall-stone disease patients without NAFLD.

\section{REFERENCES}

[1] Shaffer EA. Gallstone disease: Epidemiology of gallbladder stone disease. Best Pract Res Clin Gastroenterol 2006;20 (6):981-96.

[2] Fracanzani AL, Valenti L, Russello M, et al. Gallstone disease is associated with more severe liver damage in patients with non-alcoholic fatty liver disease. PLoS One 2012;7 (7):e41183.

[3] Ashtari S, Pourhoseingholi MA, Zali MR. Non-alcoholic fatty liver disease in Asia: prevention and planning. World J Hepatol 2015;7 (13):1788-96.

[4] Saadeh S, Younossi ZM, Remer EM, et al. The utility of radiological imaging in non-alcoholic fatty liver disease. Gastroenterology 2002;123 (3):745-50.

[5] Sandrin L, Forquet B, Hasquenoph JM, et al. Transient elastography: a new non-invasive method for assessment of hepatic fibrosis. Ultrasound Med Biol 2003;29 (12):1705-13.

[6] Tsai CJ, Leitzmann MF, Willett WC, et al. Prospective study of abdominal adiposity and gallstone disease in US men. Am J Clin Nutr 2004;80 (1):38-44.

[7] Everhart JE, Khare M, Hill M, et al. Prevalence and ethnic differences in gall-bladder disease in the United States. Gastroenterology 1999;117 (3):632-9. 
[8] Koller T, Kollerova J, Hlavaty T, et al. Cholelithiasis and markers of non-alcoholic fatty liver disease in patients with metabolic risk factors. Scand J Gastroenterol 2012;47 (2):197-203.

[9] Farah A, Qamaruddin B, Zohid AM, et al. An observational study on the association of non-alcoholic fatty liver disease and metabolic syndrome with gall stones disease requiring cholecystectomy. Annals Med \& Surg (Lond) 2017;17:7-13.
[10] Chen LY, Qiao QH, Zhang SC, et al. Metabolic syndrome and gallstone disease. World J Gastroenterol 2012;18(31):4215-20.

[11] Kratzer W, Kachele V, Mason RA, et al. Gallstone prevalence in relation to smoking, alcohol, coffee consumption and nutrition. The Ulm Gallstone Study. Scand J Gastroenterol 1997;32 (9):953-8.

[12] Browning JD, Szczepaniak LS, Dobbins R, et al. Prevalence of hepatic steatosis in an urban population in the United States: impact of ethnicity. Hepatology 2004;40

(6):1387-95 